\title{
Detection and quantification of anti-rabies glycoprotein antibodies: current state and perspectives
}

\author{
Maria Celeste Rodriguez $^{1}$ (D) $\cdot$ Diego Fontana $^{1}$ (D) $\cdot$ Ernesto Garay $^{1}$ (D) $\cdot$ Claudio Prieto $^{2}$ (D) \\ Received: 26 March 2021 / Revised: 6 August 2021 / Accepted: 10 August 2021 / Published online: 27 August 2021 \\ (C) The Author(s), under exclusive licence to Springer-Verlag GmbH Germany, part of Springer Nature 2021
}

\begin{abstract}
Rabies is an ancient fatal disease with no other available treatment than post-exposure vaccination, where the bite of infected animals, mainly dogs, is the leading cause of its transmission to human beings. In this context, global vaccination campaigns of companion animals, as well as wildlife reservoirs vaccination, are key factors to achieve the "Zero by 30 " plan that pursues the eradication of dog-mediated human rabies by 2030. Rabies virus-neutralizing antibodies (VNAs) play an essential role in the disease protection, as it correlates with an adequate immune response and allows evaluating pre- or post-exposure prophylaxis efficacy. Hence, counting with reliable, accurate, and robust serological tests is of paramount importance. Currently, RFFIT and FAVN are the gold standard VNAs tests recommended by both the WHO and the OIE. Despite these methodologies are efficient and widely used, they present several drawbacks, as they are less easily to standardize and require the use of live rabies virus, containment facilities, and skilled professionals. Thus, in this review, we describe the state-of-the-art of alternative analytical methodologies currently available for rabies serology, with novel approaches based on pseudotyped recombinant viruses and emphasizing in the antigen binding methodologies that detect and quantify antibodies against the rabies glycoprotein. We discussed the wide range of assays that are interesting tools for a faster measurement of anti-rabies glycoprotein antibodies and, in some cases, less complex and more versatile than the gold standard methods. Finally, we discussed the key issues during the design and optimization steps of ELISA assays, highlighting the importance of validation and standardization procedures to improve rabies serology tests and, as a consequence, their results.
\end{abstract}

\section{Key points}

- An exhaustive revision of rabies serology testing was made.

- No rabies serology assay can be thought as better than others for all intents and purposes.

- The validation procedure guarantees reliable and consistent results among the globe.

Keywords Rabies $\cdot$ Serology test $\cdot$ Glycoprotein $\cdot$ Neutralizing antibodies

Maria Celeste Rodriguez and Diego Fontana contributed equally to this work.

Diego Fontana

dfontana@fbcb.unl.edu.ar

1 UNL, CONICET, FBCB (School of Biochemistry and Biological Sciences), CBL (Biotechnological Center of Litoral), Cell Culture Laboratory, Ciudad Universitaria, Ruta Nacional 168 - Km 472.4 - C. C. 242 - (S3000ZAA), Santa Fe, Argentina

2 UNL, FBCB (School of Biochemistry and Biological Sciences), CBL (Biotechnological Center of Litoral), Biotechnological Development Laboratory, Ciudad Universitaria, Ruta Nacional 168 Km 472.4 - C.C. 242 - (S3000ZAA), Santa Fe, Argentina

\section{Introduction}

Throughout human history, zoonoses have become one of the greatest challenges to public health. Globally, it is estimated that about one billion cases of severe illnesses and millions of deaths occur every year from zoonoses. It is not a coincidence that the COVID-19 pandemic broke out as a result of altering environmental conditions for wildlife and the exploitation of natural resources to satisfy the demands of the population in constant growth. In particular, rabies is one of the most lethal zoonoses in the world, with a mortality approaching $100 \%$. This disease is caused by a virus of the Rhabdoviridae family, Lyssavirus genus, provoking between 40,000 and 60,000 
human deaths per year in over 150 countries, with $95 \%$ of cases occurring in Africa and Asia (World Health Organization 2018). The leading mechanism of transmission is by an animal bite, throughout the saliva of the infected host. Firstly, the virus infects peripheral nerves and, then, moves to the central nervous system. However, while rabies is fatal once symptoms appear, it is preventable due to the relatively long incubation period between virus entry and development of clinical disease (Johnson et al. 2010; Ives et al. 2019). Thus, rabies post-exposure prophylaxis (PEP), together with domestic animals and wildlife reservoir vaccination and human preexposure prophylaxis (PREP), represents the main strategies to mitigate and control rabies disease (Ives et al. 2019) (Fig. 1).

Rabies immune response involves both cell-mediated and humoral immunity; however, it is recognized that virus-neutralizing antibodies (VNAs) are critical for protection against rabies virus (RABV) infection (Katz et al. 2017). RABV is an enveloped virus, and the glycoprotein $(\mathrm{G})$ is anchored on the viral membrane. The $\mathrm{G}$ is the only surface viral protein and the only viral product known to be capable of eliciting the production of VNAs (Flamand et al. 1993; Fallahi 2005; Schnell et al. 2010). The VNAs interact with the virus, blocking their entrance to the cell, preventing (or mitigating) the course of the infection. Therefore, measure of VNAs levels is of paramount importance (Johnson et al. 2010; Moore et al. 2017; Zhao et al. 2019). Rabies serology tests are invaluable tools for determining the acquired immune response after infection and the effectiveness of vaccination campaigns of humans and wildlife (Moore et al. 2013; Moore et al. 2017; Moore and Gordon 2020).

Virus neutralization assays (VNAs) are cell-based or in vivo assays that detect the functional activity of Abs in the serum, plasma, or cerebrospinal fluid against live virus. The first assay developed to quantify rabies VNAs was the mouse neutralization test (MNT) (Webster and Dawson 1935), an in vivo assay where mice are intracerebrally inoculated with active RABV, previously incubated with diluted serum samples. The level of VNAs in the sample is evidenced by the survival of inoculated animals, where a higher percentage of survival is related to a higher VNAs titer. Because of the technical complexity, the difficulty to standardize the assay involving the use of live animals, and the growing tendency to substitute the animal use ( $3 \mathrm{R}$ principle), it has been replaced in many laboratories around the world with some exceptions in developing countries (World Health Organization 2018).
Fig. 1 Rabies virus infection and the importance of pre-exposure prophylaxis (PREP) and postexposure prophylaxis (PEP) in rabies control and prevention. Both PREP and PEP are mainly based on vaccination of individuals in order to induce an immune response where antibodies play a central role for the control of the infection. Besides, human rabies immunoglobulins (HRIGs) are frequently administered in PEP protocols

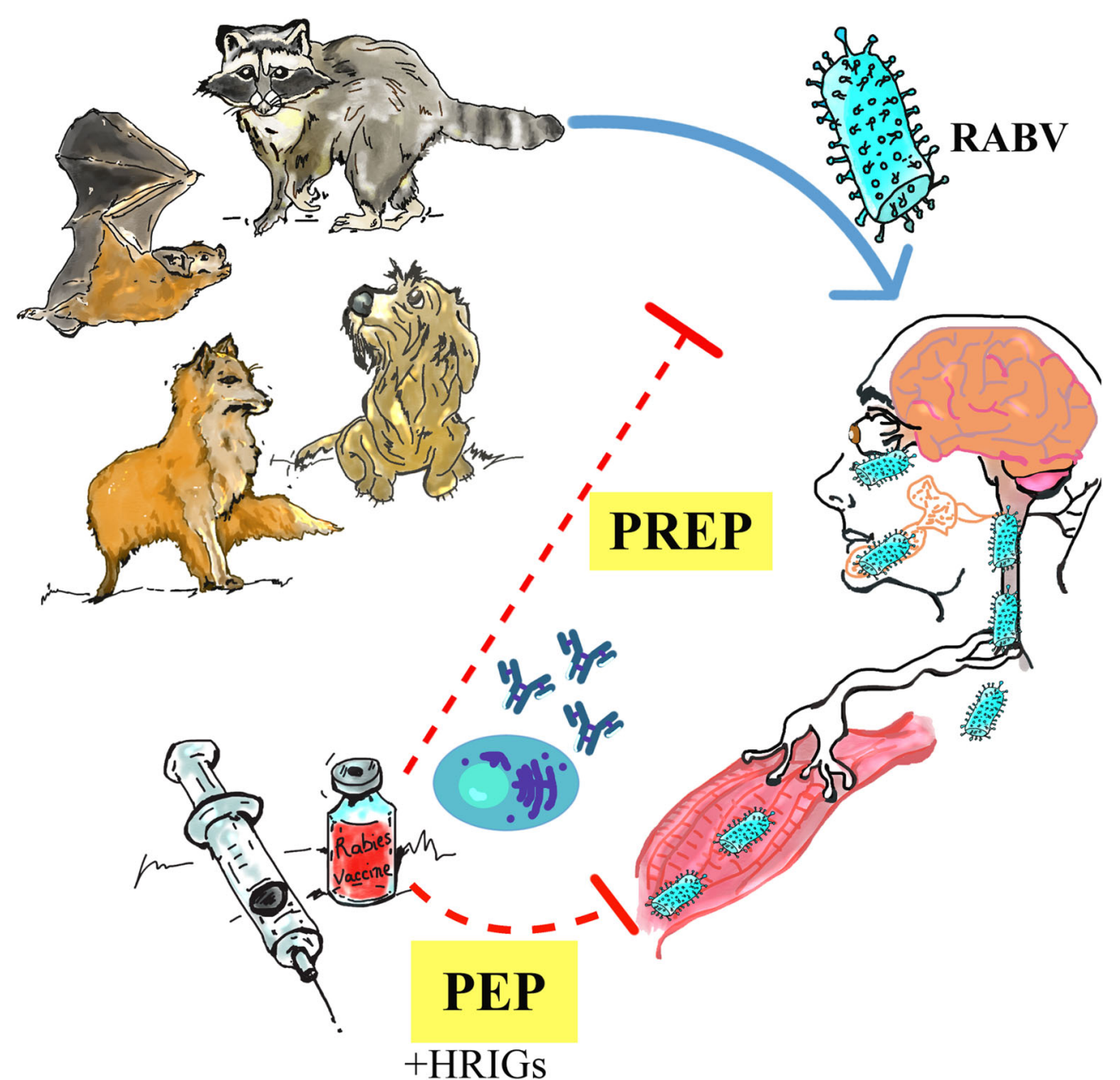


To date, only two rabies VNAs tests are considered as the gold standard methods for rabies serology and recommended by the World Health Organization (WHO) and the World Organization for Animal Health (OIE): the RFFIT (rapid fluorescent focus inhibition test) (Smith et al. 1973) and the FAVN (fluorescent antibody virus neutralization) test (Cliquet et al. 1998). These methods were designed to mimic the in vivo assay, using BHK-21 cells, which are highly susceptible to RABV infection (Fig. 2). Result interpretation is the main difference between both assays: in the former, the number of infected focus per serum dilution is used to calculate the $\mathrm{ED}_{50}(50 \%$ effective dose) neutralization titer and converted to $\mathrm{IU} / \mathrm{mL}$ if a reference standard is included in the assay (World Health Organization 2018) (Fig. 2A), whereas the latter is an "all or nothing" approach. Here, BHK-21 cells are cultured on 96-well plates instead of the 8-well chambers used on RFFIT assay; thereafter, each well is marked as positive or negative depending on the presence of infected cells on it, thereby increasing the accuracy and the quality of the test results (World Health Organization 2018) (Fig. 2B). These VNAs tests exhibit several drawbacks, as they are less easily standardized and require the use of live RABV, containment facilities, and skilled professionals, among others. Therefore, there is a need to develop alternative techniques to assess rabies control and surveillance.

Hence, alternative methods with greater assurance of consistent results and less time-consuming, as ELISA assays, are nowadays considered as acceptable to detect anti-rabies
Fig. 2 Gold standard serum neutralization tests. A RFFIT. Previously diluted serum samples are mixed with a fix amount of RABV (CVS 11 strain) in 8-well chambers (1). Following the neutralization period, a suspension of BHK cells is added and incubated for 20-24 h (2). After a wash and fix step (3), FITC conjugated antiRABV Abs are employed as the detection system (4). Results are assessed using a fluorescence microscope (5). The presence of fluorescence in the cells correlates with non-neutralized RABV (6). The $\mathrm{ED}_{50}$ neutralization titer is defined as the dilution at which $50 \%$ of the observed microscopic field contains one or more infected cells (7). B FAVN. Serially diluted serum samples are mixed with a fixed amount of RABV (CVS 11 strain) in 96-well microplates (1).

Following the neutralization period, a suspension of BHK cells is added and incubated for $48 \mathrm{~h}$ (2). After a wash and fix step (3), FITCconjugated anti-RABV Abs are employed as the detection system (4). Results are assessed using a fluorescence microscope (5). The well is considered negative if no signal is observed. By the contrary, if one or more fluorescent cells were observed, the well is considered as positive (6). Thereafter, the $\mathrm{ED}_{50}$ neutralization titer is calculated (7)

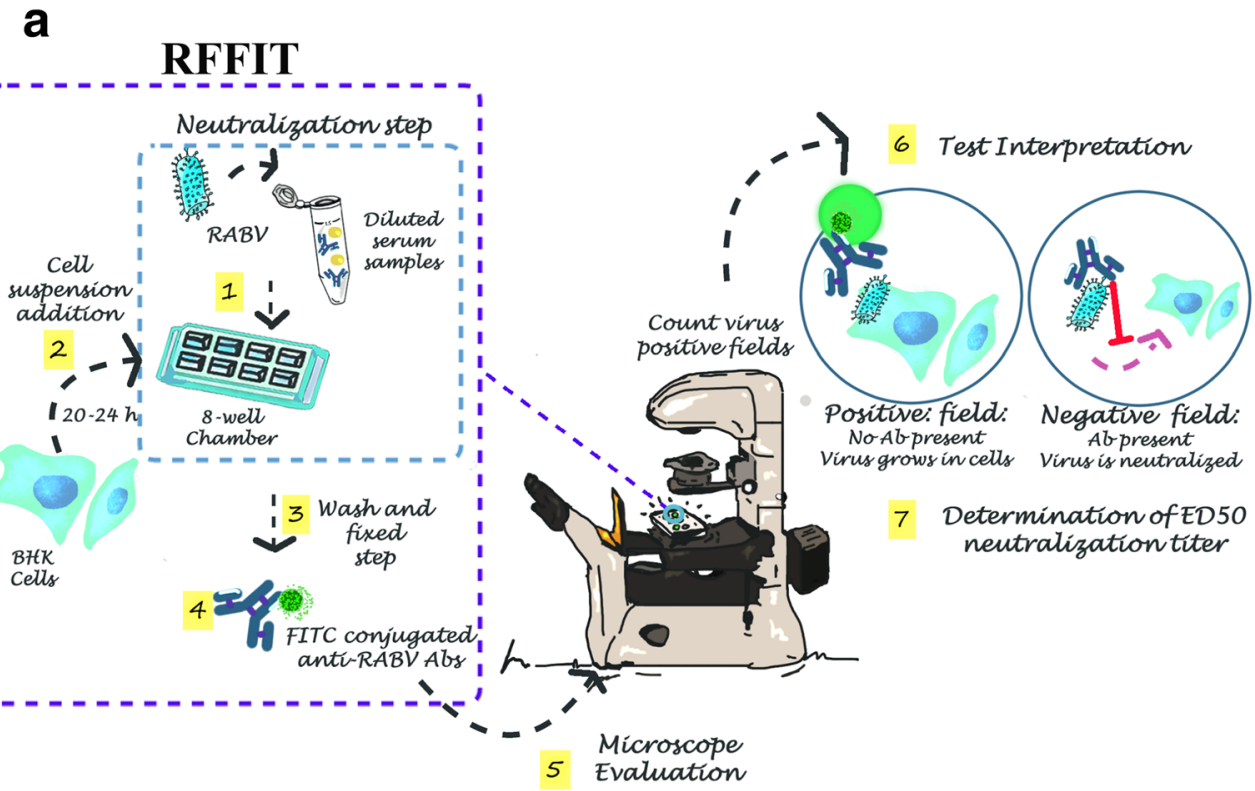

b

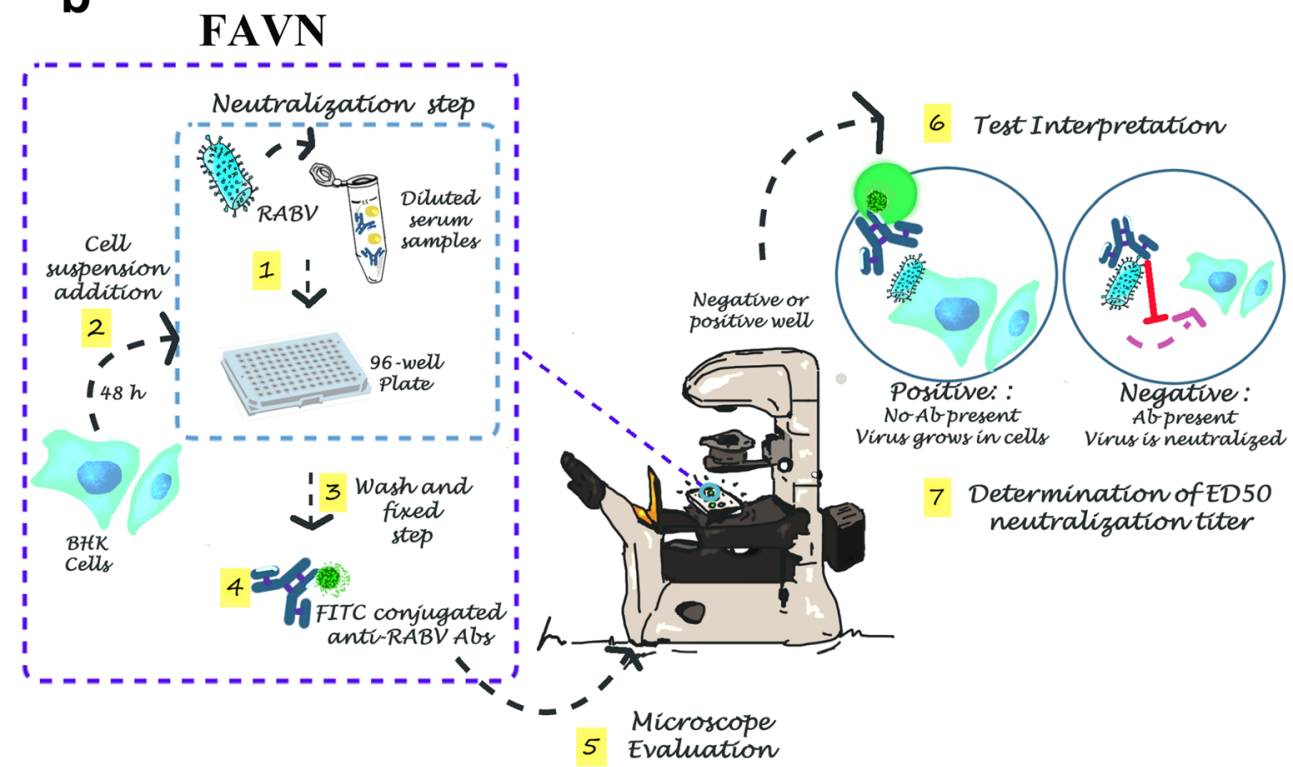


glycoprotein antibodies (Abs). Indeed, there are several works that demonstrated a good correlation between these assays and VNAs tests, based only on detecting and measuring anti-G protein antibodies (Welch et al. 2009; Wasniewski et al. 2016; Moore et al. 2017). Thus, in this review, we describe the state-of-the-art of the analytical methodologies currently available for rabies serology as an alternative to gold standard tests, taking into account other novel recombinant VNAs test approaches and the wide range of antigen binding assays. These are interesting tools for a faster measurement of anti-rabies glycoprotein antibodies and, in some cases, less complex and more versatile than the gold standard methods. However, before selecting the most suitable method, it is essential to define the fit-for-purpose of the assay.

\section{Alternative for the measurement of VNAs: pseudotyped neutralization assays}

In the last decades, recombinant viral vectors pseudotyped with a heterologous glycoprotein have been widely used for the establishment of safe and versatile serology tests. Using these vectors for the detection of VNAs (in vitro or in vivo), multiple strains or genotypes of viruses can be evaluated in a safer way. The recombinant vectors are normally produced by transient transfection methods, and the obtained viral particles are not replicative; thus, lower biohazard practices are needed (Ferrara and Temperton 2018). Lentiviruses are the most common recombinant vectors used for this purpose, being applied for a variety of enveloped viruses such as Ebola, MERS, Hepatitis C, influenza, and, more recently, for SARS-CoV-2. The envelope protein, the core and its genome, containing a reporter, is the backbone of the pseudotyped system. Several reporters are employed in the pseudotyped system being the fluorescent proteins (more often GFP) and luciferase the most commonly used reporter, due to its higher sensitivity and versatility, enabling high-throughput screening. Thereafter, the signal is measured using a fluorescent microscope or a flow cytometer for the former and a luminometer for the latter, where a cell lysis step prior to acquisition is necessary (Temperton et al. 2005; Fontana et al. 2015; Nie et al. 2017; Crawford et al. 2020; Nie et al. 2020; Yang et al. 2020) (Fig. $3)$. To guarantee comparable results among assays, titers should be reported as median tissue culture infection dose (TCID50). Besides, it is important to established the producer cell line (more often HEK293T), the transfection method (chemical or physical), culture conditions, and the harvest time, to standardize the LV production protocol assessing consistent LV titers and as a consequence, consistence, accurate, and reproducibly results.

For rabies, these recombinant viruses are pseudotyped with the G protein as the envelope protein (Fig. 3). Wright et al. described a strong correlation between FAVN and the pseudotyped assay, obtaining a specificity of $100 \%$ and a sensitivity of $94.4 \%$ (Wright et al. 2008; Wright et al. 2009). Later, Nie et al. developed a highly efficient rabies pseudotyped lentivirus production platform and a robust in vitro and in vivo neutralization assays for the evaluation of rabies vaccine. They compared these methods with current RFFIT test, showing a good linear correlation between the two assays $\left(R^{2}=0.946,<0.001\right)$ but demonstrating better reproducibility for the pseudotyped assay (Nie et al. 2017).

\section{Antigen binding assays}

Besides VNAs test, other types of methodologies commonly denominated antigen $(\mathrm{Ag})$ binding assays have been developed for rabies serology. In these assays, the Abs present in serum, plasma, or other body fluids are evaluated by its ability to bind to the rabies $\mathrm{G}$ protein, either purified from the whole virus, recombinantly expressed, or using peptides that represent epitopes exhibited in the $G$ protein amino acid sequence. A variety of methods have been developed and validated for its use in serology testing (Table 1).

Nowadays, ELISA assays are considered as the more suitable alternative approach for rabies serology, providing consistent results in several laboratories, particularly for quantitation of Abs titer in PEP and seroprevalence surveys in different wildlife reservoirs. Also, ELISA could be used to quantify human anti-rabies Abs titers following vaccination (Cliquet et al. 2004; Wasniewski et al. 2016). There are several types of ELISA used in serological methods, which vary in the antigen-antibody detection format. Besides, there are several detection systems, as biotin-avidin system conjugated to HRP, a secondary antibody conjugated to HRP, or a bacterial protein $\mathrm{A} / \mathrm{G}$ conjugated to HRP that recognize the $\mathrm{Fc}$ portion of the Igs. That is why elucidating each step of the ELISA format can help us in interpreting the results correctly.

In the indirect ELISA (iELISA) format, the $\mathrm{Ag}$ is fixed to the solid phase in the first step of the assay, and, later, the incubation of samples (that contains the Rabies Igs) allows the Ag-Ab interaction. The detection systems usually involve a secondary antibody conjugated to the HRP enzyme. This secondary antibody can be species specific which may limit the assay versatility (Cliquet et al. 2000; Cliquet et al. 2003; Zhao et al. 2019), Igs specific (more often anti-IgM or anti-IgG) (Zajac 2019), or recognizing the Fc portion of the Abs, as the protein A/G system conjugated to HRP is employed (De Toledo Piza et al. 1999; Cliquet et al. 2004; Feyssaguet et al. 2007). The main advantage of the latter detection system is that the method can be applied to samples of different species. However, the protein $\mathrm{A} / \mathrm{G}$ does not bind with the same affinity to all Igs subtypes, and it also varies between species; indeed, it is known that protein A binds preferentially to $\operatorname{IgM}$ or $\operatorname{IgG}$, but not to $\operatorname{IgG} 3$ subclass 


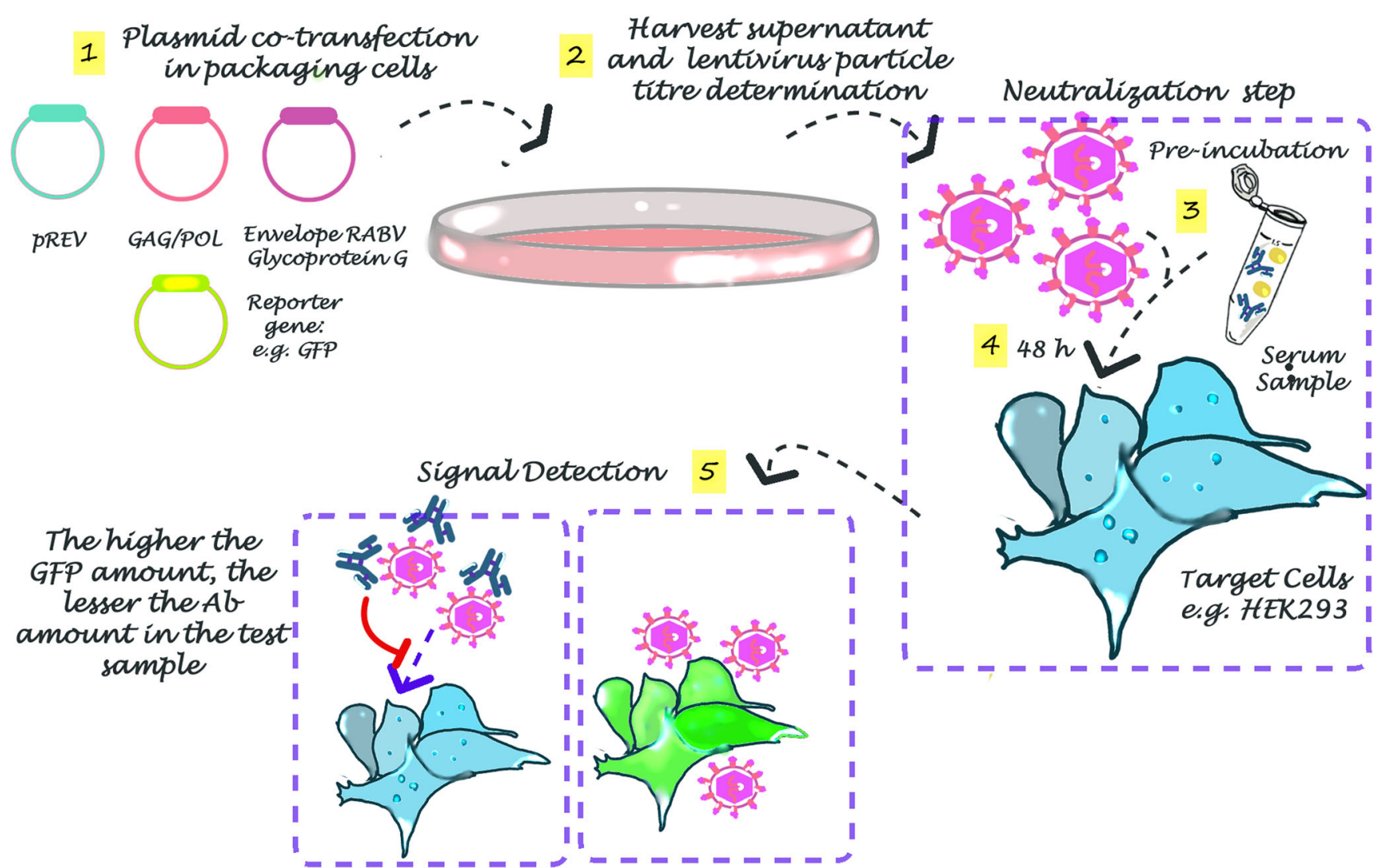

Fig. 3 Schematic representation of a lentivirus pseudotyped assay, employing GFP as reporter gene. First, packaging cells are cotransfected with the lentiviral structural plasmids and with the transfer vector containing the reporter gene. A RABV glycoprotein expressing plasmid is used to pseudotype the recombinant viruses (1). Forty-eight hours post transfection, culture supernatants containing the lentivirus are harvested and titrated (2). Thereafter, a known amount of lentiviral particles are pre-incubated with diluted serum samples (3). Later, the mix is added to a target cell culture and incubated for $48 \mathrm{~h}$ (4). Finally, the GFP signal is measured by flow cytometry (5)
(Bergmann-Leitner et al. 2008). Hence, there could be some variations in the Abs quantitation (or detection) depending on the sample (type, timing, species); thus, special care in this kind of assay must be taken, mainly during the assay result interpretation. On the other hand, if the aim of the study is to determine the proportion of $\mathrm{IgG} / \mathrm{IgM}$ in the course of rabies infection or after rabies vaccination, the detection system employing anti-IgM and anti-IgG is highly desirable. However, if the goal is to develop a good estimator of VNAs, the latter system is not the best, as it has been established that only IgGs can neutralize and protect against RABV infection. The function of IgM may be limited due to its pentameric structure, preventing the tissue penetration of $\operatorname{IgM}$ (Katz et al. 2017).

Two commercial iELISA assays are based on HRPconjugated protein A, the SERELISA® (Rabies Ab Mono Indirect) and PLATELIA® II Rabies kit (Bio-Rad). There are several reports that compared their results with the ones of VNAs assays. The SERELISA® test was described by the OIE as a screening tool to control rabies Abs in vaccinated dogs and cats, but due to the lack of sensitivity, all negative results must be confirmed in a VNAs test (Cliquet et al. 2004). Further collaborative studies demonstrated a high rate of false positive results in several laboratories (Servat and Cliquet 2006; Wasniewski et al. 2016). Regarding the PLATELIA® Rabies II kit (certified by the OIE), Feyysaguet et al described this test as a qualitative and semi-quantitative format, which exhibited a good correlation with RFFIT test for vaccinated and unvaccinated human serum sample (Feyssaguet et al. 2007). Besides, Welch et al. (2009) described good correlation between PLATELIA® and RFFIT assay in human sera samples and better than those obtained when compared to an alternative indirect ELISA kit, DGR Rabies Virus IgG Ab ELISA (DRG International), with the RFFIT (Welch et al. 2009). Moreover, the evaluation of the PLATELIA® II (ad usum veterinarium) for PEP of domestic and wildlife carnivores revealed different results between studies. Servat et al. established a threshold value of $0.5 \mathrm{EU} / \mathrm{mL}$ (harmonized with the $0.5 \mathrm{IU} / \mathrm{mL}$ ) and validated these assays, obtaining good correlation with the FAVN test (Servat et al. 2007). By the contrary, a large-scale study on pet domestic carnivores' samples significantly differed with the previously mentioned study, establishing a new cut-off value of $0.3 \mathrm{EU} / \mathrm{mL}$ to maintain the assay sensitivity but with a reduction in its specificity. These variations between studies could be due to certain 


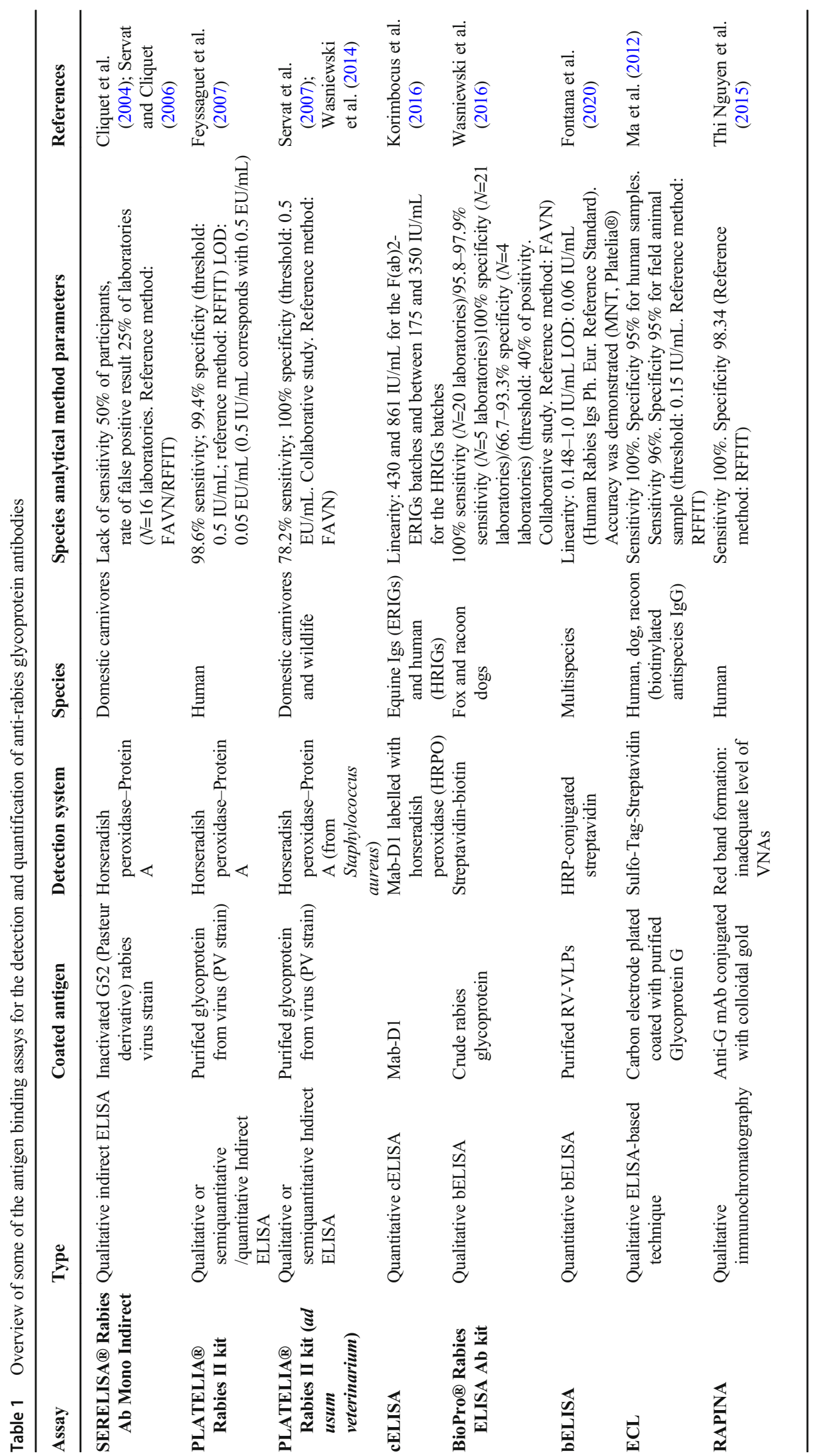


modifications during the manufacturing process of the kit (Wasniewski et al. 2014).

Competitive ELISA (cELISA) consists in fixing the Ag to the solid phase, and the Abs present in the sample compete with an enzyme-labeled $\mathrm{Ab}$ for binding to the $\mathrm{Ag}$. Thus, in this assay, the higher the signal, the lesser the concentration of $\mathrm{Ab}$ in the test sample (Zhang et al. 2009). Another alternative of this format is the one described by Korimbocus et al. for potency testing of equine anti-rabies sera, employing the Mab D1 clone that recognize one of the immunodominant epitopes of the rabies glycoprotein involves in the induction of VNAs (site III) (Jallet et al. 1999), demonstrating a good correlation with MNT assay (Korimbocus et al. 2016). They also evaluated human anti-rabies Igs, obtaining higher and more heterogeneous titers in comparison with PLATELIA ${ }^{\circledR}$ Rabies II and RFFIT.

Blocking ELISA (bELISA) is another format of ELISA assay, where the Ag is also fixed to the plate, but the Abs present in the test sample block the next step, that is, the addition of an enzyme-labeled $\mathrm{Ab}$ that binds to the $\mathrm{Ag}$ (Fig. 4). Hence, and similar to cELISA, the higher the signal, the lesser the $\mathrm{Ab}$ amount in the test sample. An example of a commercial bELISA is the BioPro Rabies ELISA Ab kit (O. K. SERVIS BioPro) that employed crude glycoprotein as coated-antigen and streptavidin-biotin as detection system. Wasniewski and Cliquet compared the BioPro performance with the one of the PLATELIA $®$ Rabies II kits (ad usum veterinarium), determining that the former exhibited better sensitivity and a good correlation with the FAVN test in serum samples of domestic carnivores (Wasniewski and Cliquet 2011). Further studies demonstrated good correlation with FAVN in serum sample of orally vaccinated fox and raccoon dogs (Wasniewski et al. 2013). Besides, BioPro ELISA was further validated throughout an international study involving serum samples from foxes and raccoon dogs vaccinated with different vaccine strains available in Europe (Wasniewski et al. 2016). Dascalu et al. also demonstrated good correlations with the FAVN test for orally vaccinated wild boars and foxes (Dascalu et al. 2019).

Recently, our group has published a validated bELISA for multispecies serum rabies immunoglobulins quantitation employing purified rabies virus-like particles (RV-VLPs) as
Fig. 4 A schematic representation of bELISA assay. First, the antigen is immobilized in 96-well plates (1). Second, Abs present in the serum sample recognizes an epitope of the target antigen (2). Third, a biotinconjugated $\mathrm{Ab}$ interacts with the Ag not blocked by the Abs of the test sample (3). Fourth, a streptavidin-peroxidase conjugate interacts with the biotin of the conjugated $\mathrm{Ab}(4)$. The peroxidase reacts with a substrate to produce a detectable signal (5). Here, the higher the signal, the lesser the $\mathrm{Ab}$ amount in the test serum samples

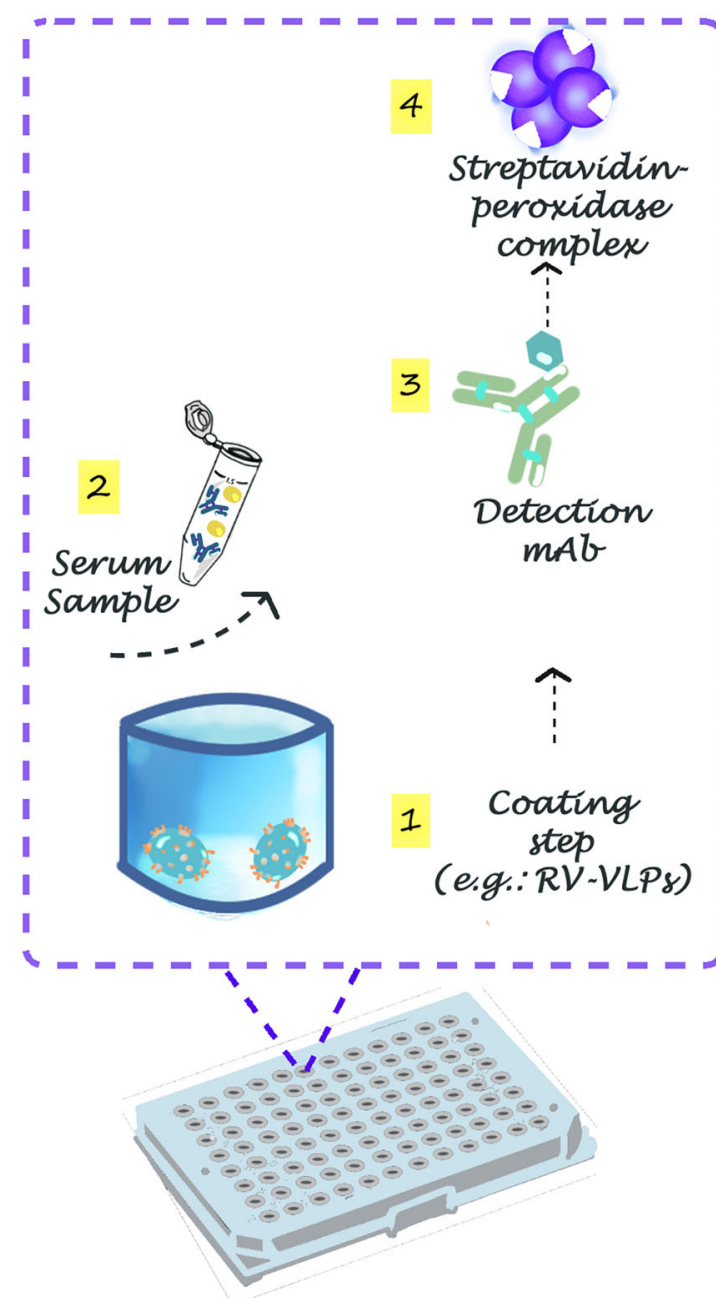

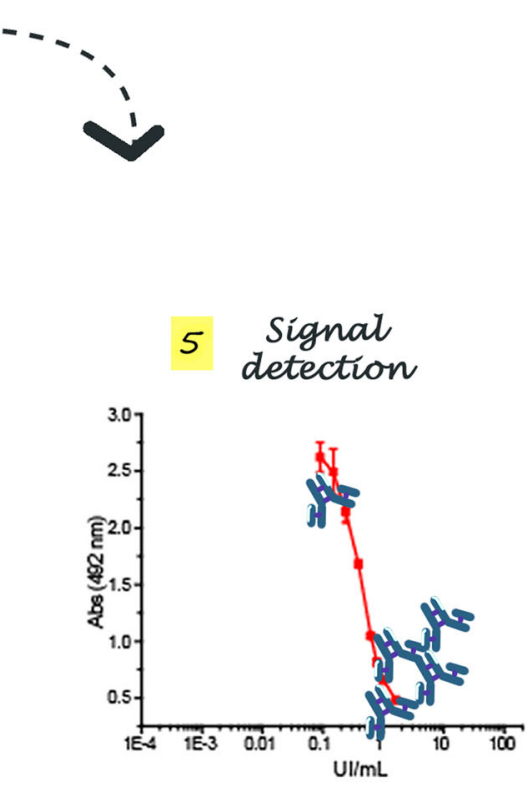

The higher the signal, the lesser the $A b$ amount in the test sample 
the coated antigen and a biotin-conjugated monoclonal Abs as detection system (Fig. 4). We demonstrated a good correlation of the bELISA with the PLATELIA® Rabies II kit and the MNT test (Fontana et al. 2020). Despite further characterization must be performed, evaluating a bigger serum sample panel, and that the assay reproducibility must be guaranteed, we postulated this assay as a potential alternative to the gold standard methods in PEP schemes and within rabies surveillance programs.

Other methods have also been developed and appeared to be promising alternatives, exhibiting good correlation with the RFFIT. For instance, an electrochemiluminescence (ECL) adaptation of a bELISA using ECL labels and carbon electrode plates to detect binding events presented higher sensitivity and faster results compared to traditional ELISA detection methods (Ma et al. 2012). Furthermore, indirect fluorescent antibody test is also a semi-quantitative test for rabies immunoglobulins detection where the presence of rabies Igs is assessed by a fluorescence signal, requiring as little as $1.5 \mathrm{~h}$ to obtain the result. However, a fluorescence microscope is needed (Messenger and Rupprecht 2015). In addition, RAPINA (rapid neutralizing antibody) test based on immunochromatography principle was proposed as a fast alternative method to detect the presence of neutralizing Abs in human serum samples (Shiota et al. 2009). The latter could be an interesting alternative technique for initial screening methods for fieldwork (Thi Nguyen et al. 2015).

\section{Design, optimization, and validation of $\mathrm{Ag}$ binding assays}

As we mentioned, the most effective strategy to control rabies and mitigate its effects are vaccinations campaigns. Hence, it is of paramount importance counting with serology assays allowing accurate and consistent results. In this context, despite ELISAs sometimes fail in the accurate estimation of the potential acquired immunity after infection or vaccination, as functional and non-functional Abs are measured, several others have proven a good correlation with the gold standard assays. Besides, we must not deny all the inherent characteristics of this technique that are a benefit in comparison with gold standard assays: safer, not requiring (in general) virus manipulation, versatility, high-throughput, high consistent results, less time, and associated cost. Finally, another point to consider is that ELISAs are flexible techniques, easily adaptable to laboratories of low-level complexity, making it a particularly interesting candidate in developing countries where rabies is endemic. Therefore, we will briefly discuss the main topics to consider during the development, optimization, and validation of ELISA assays.

When designing an assay, it should be borne in mind the "why" and the "what for" of this assay, that is, the aim and the scope of the analytical test that will guide the selection of the best ELISA platform. Briefly, the ELISA format will be conditioned by the quality and the type of input materials: (i) purity, type of antigen; (ii) type of capture (if needed) and detection antibody (polyclonal or monoclonal Abs, antigenic sites, isotypes); and (ii) detection system, that also, will influence in the sensitivity and the LOD and LOQ of the technique. Generally, fluorescence techniques exhibit a higher sensitivity than colorimetric assay, requiring less amount of the test sample. Besides, the properties of the test sample (type, source, quality, accessibility) will condition the sample treatments: for instance, the proper dilution to guarantee its detection but avoid the matrix effect or the need (or not) to implement a serum heat-inactivation protocol, avoiding proportional systematic errors.

Next, once an ELISA format has been chosen, identifying the critical step/s of the assay should be crucial and can be determined through a screening step, where many factors are studied to identify those having a significant effect on the analyzed response, throughout a design of experiment (DoE). Here, specialized knowledge and experience, together with data from literature, will facilitate the task. The coated-antigen characteristics are probably one of the most crucial steps in the ELISA assay together with the amount and type of capture/detection Abs. For instance, the use of rabies $\mathrm{G}$ protein as the coating $\mathrm{Ag}$ is more relevant when assessing the oral vaccination efficacy than the whole virus, as VNAs are primarily directed against the glycoprotein. Thus, it will be better to use the glycoprotein as the coated $\mathrm{Ag}$. Besides, the degree of purity of the coated-Ag may influence the assay selectivity. It has been shown that purified $\mathrm{Ag}$ provides higher homogeneity, lesser interfering substances, and a better evaluation of VNAs (Zhao et al. 2019). However, it has also been reported that the $\mathrm{Ag}$ purification protocols may affect the immunogenicity of the rabies $\mathrm{G}$ protein (Wasniewski and Cliquet 2011; Wasniewski et al. 2013).

Once the critical factors have been identified, the optimization step must be followed, using a multivariate approach, as an "optimization" procedure performed by OVAT (one variable at a time) does not guarantee at all that the real optimum will be hit (Leardi 2009). By the contrary, in a multivariate strategy, significant factors (at different levels) are studied simultaneously in a predefined number of experiments (Goicochea 2018). In our previous work (Fontana et al. 2020), an optimization multivariate strategy was followed which allowed us to identify the purified RV-VLPs and the rabies glycoprotein monoclonal antibody (mAb AB5) as the key components of this assay. In the RV-VLPs, the rabies $G$ is presented in high density in the surface of the particles in its trimeric form (Fontana et al. 2015). Besides, the selected detection system (streptavidin-peroxidase) influences the assay sensitivity, so it was selected as an additional variable to be optimized. Moreover, we demonstrated that the mAb AB5 recognized rabies $\mathrm{G}$ on its native state-structure, making it 
an invaluable feature for this assay, as it can recognize essential epitopes for virus neutralization.

After the optimization process of the assay, it is essential to validate and standardize the method to obtain consistent and comparable results. The goal of an assay validation is to provide assurance that the method is likely to produce results that are fit-for-purpose. Hence, we must differentiate between the aim of a qualitative and a quantitative ELISA assay. The aim of the former is to assess if the analyte (in this case, rabies Igs) is present or not in the test sample and which is the minimum level of detection (cut-off). By the contrary, in the latter assay, a limit of quantitation (LOQ) must be established, and the amount of the analyte in the test sample is determined by comparing with the reference standard's response. The validation procedure of the test will be not the same if it is a qualitative or a quantitative assay. Indeed, the extent of the validation procedure depends on the status of the assay, and it is determined by the customer needs or as laid down on regulations. For qualitative methods, it is essential to determine the sensitivity (or its inverse false negative rate) and specificity (or its inverse false positive rate) through a ROC curve, finding the optimal cut-off value with a broad spectrum of test samples and avoiding bias (Hajian-Tilaki 2013). In these types of assays, establishing the cut-off value is critical. That is, in part, because the higher the threshold value, the lesser the false positive rate and, thus, a truly guarantee of seroconversion, whereas for quantitative assay, the sensitivity, analytical sensitivity, linearity, limit of detection, LOQ, selectivity, robustness, accuracy, and precision (at least repeatability and intermediate precision) must be evaluated. In addition, the stability is also important. The use of a serum reference standard with known stability is also essential. If a collaboration study is performed, the assay's reproducibility can be determined to standardize the procedure.

As we previously mentioned, sample characteristics (e.g., matrix, stability, timing, quality, cross-reactivity Abs) are other essential aspects to consider during the validation procedure. The sample matrix can contribute or interfere with the analyte signal, compromising the assay's selectivity and accuracy. Thus, beside the employment of the corresponding blanks (reactive and sample), the matrix of the sample must be characterized and studied through a different statistical test as recovery or elliptical joint confidence region (EJCR) test (Olivieri 2015), by comparing the signal of the reference and the reference embedded in a certain matrix.

Finally, it is essential to demonstrate the correlation between the developed ELISA assay and the gold standard methods. Hence, we would like to add a final commentary: several works state a good correlation between both assays employing only the $R^{2}$ coefficient. However, Pearson's coefficient only measures the variation of one variable with respect to the other but not its degree of agreement. Hence, it does not per se provide any information and should be complemented with Bland and Atman diagram test for quantitative methods and Cohen's Kappa or Mc Neman's test for qualitative methods or another alternative method as EJCR test.

\section{Conclusions and future perspectives}

Rabies is one of the most lethal worldwide infection diseases. Tens of thousands of deaths are reported annually, but the official figures are believed to be highly underestimated (Banyard et al. 2013). In this scenario, PEP and PREP constitute the main tools in rabies control. In this context, serology is a critical component for rabies surveillance and prevention programs to mitigate its risk in human beings and in domestic and wildlife animals. Currently, RFFIT and FAVN are still being the gold standard VNAs assays for rabies serology test. However, different assays employing pseudotyped recombinant viruses represent a promising and safer alternative to the conventional methods, characterized by a good reproducibility and sensitivity performance. On the other hand, ELISA and other Ag binding assays are considered acceptable for anti-rabies Abs detection, exhibiting greater assurance of consistent results and less time consuming than VNAs tests. However, no method is better than another, and the selection will depend on its fit-for-purpose, meaning that the applied serology test can solve a specific problem and that the obtained data can be easily interpreted as a result.

Design, optimization, and validation procedures are critical to achieve both fitness of purpose and reliable serology test and, consequently, reliable results. One of the greatest challenges to improve rabies control and prevention strategies is to standardize and harmonize rabies serology results worldwide, requiring a continued collaboration between national and international laboratories, companies, and regulatory agencies.

Author contribution MCR, DF, and EG conducted the bibliography revision and wrote the manuscript. MCR performed the artwork. CP revised the manuscript. All the authors approved the manuscript.

\section{Declarations}

Ethical approval This article does not contain any studies with human participants or animals performed by any of the author.

Conflict of interest The authors declare no competing interests.

\section{References}

Banyard AC, Horton DL, Freuling C, Müller T, Fooks AR (2013) Control and prevention of canine rabies: the need for building laboratory-based surveillance capacity. Antivir Res 98:357-364. https://doi.org/10.1016/j.antiviral.2013.04.004

Bergmann-Leitner ES, Mease RM, Duncan EH, Khan F, Waitumbi J, Angov E (2008) Evaluation of immunoglobulin purification methods and their impact on quality and yield of antigen-specific 
antibodies. Malar J 7:1-10. https://doi.org/10.1186/1475-2875-7129

Cliquet F, Aubert M, Sagné L (1998) Development of a fluorescent antibody virus neutralisation test (FAVN test) for the quantitation of rabies-neutralising antibody. J Immunol Methods 212:79-87

Cliquet F, Sagné L, Schereffer JL, Aubert MF (2000) ELISA test for rabies antibody titration in orally vaccinated foxes sampled in the fields. Vaccine 18:3272-3279. https://doi.org/10.1016/S0264-410X (00)00127-4

Cliquet F, Müller T, Mutinelli F, Geronutti S, Brochier B, Selhorst T, Schereffer JL, Krafft N, Burow J, Schameitat A, Schlüter H, Aubert M (2003) Standardisation and establishment of a rabies ELISA test in European laboratories for assessing the efficacy of oral fox vaccination campaigns. Vaccine 21:2986-2993. https://doi.org/10. 1016/S0264-410X(03)00102-6

Cliquet F, McElhinney LM, Servat A, Boucher JM, Lowings JP, Goddard T, Mansfield KL, Fooks AR (2004) Development of a qualitative indirect ELISA for the measurement of rabies virusspecific antibodies from vaccinated dogs and cats. J Virol Methods 117:1-8. https://doi.org/10.1016/j.jviromet.2003.12.001

Crawford KHD, Eguia R, Dingens AS, Loes AN, Malone KD, Wolf CR, Chu HY, Tortorici MA, Veesler D, Murphy M, Pettie D, King NP, Balazs AB, Bloom JD (2020) Protocol and reagents for pseudotyping lentiviral particles with SARS-CoV-2 spike protein for neutralization assays. Viruses 12. https://doi.org/10.3390/ v12050513

Dascalu MA, Wasniewski M, Picard-Meyer E, Servat A, Daraban Bocaneti F, Tanase OI, Velescu E, Cliquet F (2019) Detection of rabies antibodies in wild boars in north-east Romania by a rabies ELISA test. BMC Vet Res 15:1-12. https://doi.org/10.1186/ s12917-019-2209-x

De Toledo Piza AS, Santos JLF, Chaves LB, Zanetti CR (1999) An ELISA suitable for the detection of rabies virus antibodies in serum samples from human vaccinated with either cell-culture vaccine or suckling-mouse-brain vaccine. Rev. Inst. Med. Trop. Sao Paulo 41: $39-43$

Fallahi F (2005) Characterization of epitopes on the rabies virus glycoprotein by selection and analysis of escape mutants. Virus Res 220: 161-171. https://doi.org/10.1016/j.virusres.2016.04.019

Ferrara, Temperton (2018) Pseudotype neutralization assays: from laboratory bench to data analysis. Methods Protoc 1:8. https://doi.org/10. 3390/mps 1010008

Feyssaguet M, Dacheux L, Audry L, Compoint a, Morize JL, Blanchard I, Bourhy H (2007) Multicenter comparative study of a new ELISA, PLATELIA RABIES II, for the detection and titration of anti-rabies glycoprotein antibodies and comparison with the rapid fluorescent focus inhibition test (RFFIT) on human samples from vaccinated and non-vacci. Vaccine 25:2244-2251. https://doi.org/10.1016/j. vaccine.2006.12.012

Flamand A, Raux H, Gaudin Y, Ruigrok RBH (1993) Mechanisms of rabies virus neutralization. Virology 194:302-313. https://doi.org/ 10.1006/viro.1993.1261

Fontana D, Kratje R, Etcheverrigaray M, Prieto C (2015) Immunogenic virus-like particles continuously expressed in mammalian cells as a veterinary rabies vaccine candidate. Vaccine 33:4238-4246. https:// doi.org/10.1016/j.vaccine.2015.03.088

Fontana D, Rodriguez MC, Garay E, Russo S, Prieto C (2020) Optimization and validation of a blocking ELISA for quantitation of anti-rabies immunoglobulins in multispecies sera. Appl Microbiol Biotechnol 104:4127-4139. https://doi.org/10.1007/ s00253-020-10490-6

Goicochea H (2018) Experimental design. In: Nollet L, Siddiqi and K (eds) Fingerprinting techniques in food authentication and traceability. pp 187-206
Hajian-Tilaki K (2013) Receiver operating characteristic (ROC) curve analysis for medical diagnostic test evaluation. Casp J Intern Med 4:627-635

Ives A, Dieuzy-Labaye I, Abela-Ridder B (2019) Global characteristics of the rabies biologics market in 2017. Vaccine 37:A73-A76. https://doi.org/10.1016/j.vaccine.2018.10.012

Jallet C, Jacob Y, Bahloul C, Drings A, Desmezieres E, Tordo N, Perrin P (1999) Chimeric lyssavirus glycoproteins with increased immunological potential. J Virol 73:225-233

Johnson N, Cunningham AF, Fooks AR (2010) The immune response to rabies virus infection and vaccination. Vaccine 28:3896-3901. https://doi.org/10.1016/j.vaccine.2010.03.039

Katz ISS, Guedes F, Fernandes ER, dos Ramos SS (2017) Immunological aspects of rabies: a literature review. Arch Virol 162:3251-3268. https://doi.org/10.1007/s00705-017-3484-0

Korimbocus J, Dehay N, Tordo N, Cano F, Morgeaux S (2016) Development and validation of a quantitative competitive ELISA for potency testing of equine anti rabies sera with other potential use. Vaccine 34:3310-3316. https://doi.org/10.1016/j.vaccine. 2016.04.086

Leardi R (2009) Experimental design in chemistry: a tutorial. Anal Chim Acta 652:161-172. https://doi.org/10.1016/j.aca.2009.06.015

Ma X, Niezgoda M, Blanton JD, Recuenco S, Rupprecht CE (2012) Evaluation of a new serological technique for detecting rabies virus antibodies following vaccination. Vaccine. 30:5358-5362. https:// doi.org/10.1016/j.vaccine.2012.06.037

Messenger S, Rupprecht CE (2015) An indirect fluorescent antibody test for the serological detection of rabies virus immunoglobulin $\mathrm{G}$ and immunoglobulin M antibodies. Second Edi, Elsevier Inc

Moore SM, Gordon CR, Chapter 13 - Measures of rabies immunity, Editor(s): Anthony R. Fooks, Alan C. Jackson, Rabies (Fourth Edition), Academic Press, 2020, Pages 445-479, https://oi.org/10. 1016/B978-0-12-818705-0.00013-3

Moore SM, Gordon CR, Hanlon CA (2013) Chapter 12 - Measures of rabies immunity. Editor(s): Alan C. Jackson, Rabies (Third Edition), Academic Press. Pages 461-495, https://doi.org/10.1016/B978-012-396547-9.00021-3

Moore SM, Gilbert A, Vos A, Freuling CM, Ellis C, Kliemt J, Müller T (2017) Rabies virus antibodies from oral vaccination as a correlate of protection against lethal infection in wildlife. Trop Med Infect Dis 2. https://doi.org/10.3390/tropicalmed2030031

Nie J, Wu X, Ma J, Cao S, Huang W, Liu Q, Li X, Li Y, Wang Y (2017) Development of in vitro and in vivo rabies virus neutralization assays based on a high-titer pseudovirus system. Sci Rep 7:1-12. https://doi.org/10.1038/srep42769

Nie J, Li Q, Wu J, Zhao C, Hao H, Liu H, Zhang L, Nie L, Qin H, Wang M, Lu Q, Li X, Sun Q, Liu J, Fan C, Huang W, Xu M, Wang Y (2020) Quantification of SARS-CoV-2 neutralizing antibody by a pseudotyped virus-based assay. Nat Protoc 15:3699-3715. https:// doi.org/10.1038/s41596-020-0394-5

Olivieri AC (2015) Practical guidelines for reporting results in single- and multi-component analytical calibration: a tutorial. Anal Chim Acta 868:10-22. https://doi.org/10.1016/j.aca.2015.01.017

Schnell MJ, McGettigan JP, Wirblich C, Papaneri A (2010) The cell biology of rabies virus: using stealth to reach the brain. Nat Rev Microbiol 8:51-61. https://doi.org/10.1038/nrmicro2260

Servat A, Cliquet F (2006) Collaborative study to evaluate a new ELISA test to monitor the effectiveness of rabies vaccination in domestic carnivores. Virus Res 120:17-27. https://doi.org/10.1016/j.virusres. 2006.02.011

Servat A, Feyssaguet M, Blanchard I, Morize JL, Schereffer JL, Boue F, Cliquet $F$ (2007) A quantitative indirect ELISA to monitor the effectiveness of rabies vaccination in domestic and wild carnivores. $\mathrm{J}$ Immunol Methods 318:1-10. https://doi.org/10.1016/j.jim.2006.07. 026 
Shiota S, Mannen K, Matsumoto T, Yamada K, Yasui T, Takayama K, Kobayashi Y, Khawplod P, Gotoh K, Ahmed K, Iha H, Nishizono A (2009) Development and evaluation of a rapid neutralizing antibody test for rabies. J Virol Methods 161:58-62. https://doi.org/10.1016/j. jviromet.2009.05.018

Smith JS, Yager PA, Baer GM (1973) A rapid reproducible test for determining rabies neutralizing antibody. Bull World Health Organ 48:535-541

Temperton NJ, Chan PK, Simmons G, Zambon MC, Tedder RS, Takeuchi Y, Weiss RA (2005) Longitudinally profiling neutralizing antibody response to SARS coronavirus with pseudotypes. Emerg Infect Dis 11:411-416. https://doi.org/10.3201/eid1103.040906

Thi Nguyen KA, Nguyen TT, Nguyen DV, Ngo GC, Nguyen CN, Yamada K, Noguchi K, Ahmed K, Hoang HD, Nishizono A (2015) Evaluation of rapid neutralizing antibody detection test against rabies virus in human sera. Trop Med Health 43:111-116. https://doi.org/10.2149/tmh.2014-35

Wasniewski M, Cliquet F (2011) Evaluation of ELISA for detection of rabies antibodies in domestic carnivores. J Virol Methods 179:166175. https://doi.org/10.1016/j.jviromet.2011.10.019

Wasniewski M, Guiot AL, Schereffer JL, Tribout L, Mähar K, Cliquet F (2013) Evaluation of an ELISA to detect rabies antibodies in orally vaccinated foxes and raccoon dogs sampled in the field. J Virol Methods 187:264-270. https://doi.org/10.1016/j.jviromet.2012.11. 022

Wasniewski M, Labbe A, Tribout L, Rieder J, Labadie A, Schereffer JL, Cliquet F (2014) Evaluation of a rabies ELISA as an alternative method to seroneutralisation tests in the context of international trade of domestic carnivores. J Virol Methods 195:211-220. https://doi.org/10.1016/j.jviromet.2013.10.021

Wasniewski M, Almeida I, Baur A, Bedekovic T, Boncea D, Chaves LB, David D, De Benedictis P, Dobrostana M, Giraud P, Hostnik P, Jaceviciene I, Kenklies S, König M, Mähar K, Mojzis M, Moore S, Mrenoski S, Müller T et al (2016) First international collaborative study to evaluate rabies antibody detection method for use in monitoring the effectiveness of oral vaccination programmes in fox and raccoon dog in Europe. J Virol Methods 238:77-85. https://doi.org/ 10.1016/j.jviromet.2016.10.006

Webster LT, Dawson JR (1935) Early diagnosis of rabies by mouse inoculation. Measurement of humoral immunity to rabies by mouse protection test. Proc Soc Exp Biol Med, 32:570
Welch RJ, Anderson BL, Litwin CM (2009) An evaluation of two commercially available ELISAs and one in-house reference laboratory ELISA for the determination of human anti-rabies virus antibodies. $\mathrm{J}$ Med Microbiol 58:806-810. https://doi.org/10.1099/jmm.0. 006064-0

World Health Organization. (2018). WHO expert consultation on rabies: third report. World Health Organization. https://apps.who.int/iris/ handle/10665/272364

Wright E, Temperton NJ, Marston DA, LM ME, Fooks AR, Weiss RA (2008) Investigating antibody neutralization of lyssaviruses using lentiviral pseudotypes: a cross-species comparison. J Gen Virol 89:2204-2213. https://doi.org/10.1099/vir.0.2008/000349-0

Wright E, McNabb S, Goddard T, Horton DL, Lembo T, Nel LH, Weiss RA, Cleaveland S, Fooks AR (2009) A robust lentiviral pseudotype neutralisation assay for in-field serosurveillance of rabies and lyssaviruses in Africa. Vaccine 27:7178-7186. https://doi.org/10. 1016/j.vaccine.2009.09.024

Yang R, Huang B, A R, Li W, Wang W, Deng Y, Tan W (2020) Development and effectiveness of Pseudotyped SARS-CoV-2 system as determined by neutralizing efficiency and entry inhibition test in vitro. Biosaf Heal:4-8. https://doi.org/10.1016/j.bsheal.2020.08. 004

Zajac MD (2019) Development and evaluation of a rabies enzyme-linked immunosorbent assay ( ELISA ) targeting IgM and $\operatorname{IgG}$ in human sera by. https://doi.org/10.13140/RG.2.2.32365.54242

Zhang S, Liu Y, Zhang F, Hu R (2009) Competitive ELISA using a rabies glycoprotein-transformed cell line to semi-quantify rabies neutralizing-related antibodies in dogs. Vaccine 27:2108-2113. https://doi.org/10.1016/j.vaccine.2009.01.126

Zhao R, Yu P, Shan Y, Thirumeni N, Li M, Lv Y, Li J, Ren W, Huang L, Wei J, Sun Y, Zhu W, Sun L (2019) Rabies virus glycoprotein serology ELISA for measurement of neutralizing antibodies in sera of vaccinated human subjects. Vaccine 37:6060-6067. https://doi. org/10.1016/j.vaccine.2019.08.043

Publisher's note Springer Nature remains neutral with regard to jurisdictional claims in published maps and institutional affiliations. 